\title{
Analysis of stationary droplets in a generic Turing reaction-diffusion system
}

\author{
Thomas E. Woolley* and Ruth E. Baker \\ Centre for Mathematical Biology, Mathematical Institute, University of Oxford, 24-29 St. Giles', Oxford OX1 3LB, United Kingdom \\ Philip K. Maini \\ Centre for Mathematical Biology, Mathematical Institute, University of Oxford, 24-29 St. Giles', Oxford OX1 3LB, United Kingdom \\ and Oxford Centre for Integrative Systems Biology, Department of Biochemistry, University of Oxford, South Parks Road, \\ Oxford OX1 3QU, United Kingdom \\ José Luis Aragón \\ Centro de Física Aplicada y Tecnologia Avanzada, UNAM, Apartado Postal 1-1010, 76000 Queretaro, Mexico
}

Rafael A. Barrio

Instituto de Física, UNAM, Apartado Postal 20-364, 01000 México, D.F., Mexico

(Received 21 July 2010; revised manuscript received 6 October 2010; published 23 November 2010)

\begin{abstract}
Solitonlike structures called "droplets" are found to exist within a paradigm reaction-diffusion model that can be used to describe patterning in a number of biological systems, for example, on the skin of various fish species. They have also been found in many other systems that can be modeled with a complex GinzburgLandau system. These droplets can be analyzed in the biological paradigm model because the system has two nonzero stable steady states that are symmetric; however, the asymmetric case is more challenging. We first review the properties of the paradigm system and then extend a recently developed perturbation technique [D. Gomila et al., J. Opt. B: Quantum Semiclassical Opt. 6, S265 (2004)] to investigate the weakly asymmetric case. We compare the results of our mathematical analysis with numerical simulations and show good agreement in the region where the assumptions hold.
\end{abstract}

DOI: 10.1103/PhysRevE.82.051929

PACS number(s): 87.18.Hf, 87.17.Pq, 87.17.Aa, 87.10.Tf

\section{INTRODUCTION}

The study and analysis of patterning in reaction-diffusion equations has recently had a resurgence due to increased experimental evidence of their existence. Turing patterns in the chlorite-iodide-malonic acid [1] and chlorite dioxide-iodidemalonic acid [2] reactions were discovered nearly 40 years after their existence was postulated by Alan Turing in 1952 [3]. Although the number of actual chemical reactions which produce these patterns is small, the idea of diffusion-driven instability, extended by Gierer and Meinhardt [4] to the paradigm-shifting patterning principle of short-range activation and long-range inhibition, has stimulated much biological research in pattern formation. The mechanism has been used to describe many different phenomena, such as pigmentation patterning in the angelfish Pomacanthus imperator [5], the pattern of follicles on the skin of mice [6], the spot pattern of lady bugs [7], and the coloring of the shells of marine snails [8].

We have proposed a general, yet simple, reactiondiffusion system [Eqs. (3) and (4)] [referred to as the BarrioVarea-Argón-Maini (BVAM) model from now on], which presents a rich bifurcation structure and a variety of patterns which makes it a very versatile system for modeling biological phenomena [9]. It can produce not only Turing patterns but also Hopf bifurcations, Turing-Hopf bifurcations, limit cycles, and wave front profiles. As a result of interactions

\footnotetext{
*woolley@maths.ox.ac.uk
}

between the various different bifurcation structures the BVAM model can give qualitative agreement with the complicated patterns found on the skin of the freshwater catfish Pseudoplatystoma fasciatum (Surubim pintado), P. tigrinum (Surubim atigrado), and $P$. coruscans (Surubim manchado) by a simple change of one parameter [10]. Further, by coupling two BVAM systems together with different temporal and spatial scales it has been shown that patterns similar to the pigmentation patterns on the skin of the stingray Potamotrygon motoro can be produced (see Fig. 1) [10].

The spotted pattern observed on the skin of stingrays is found within the bistable region of the BVAM model, and it is this situation which is of interest in this paper. The spots are actually large stable domain walls separating the two
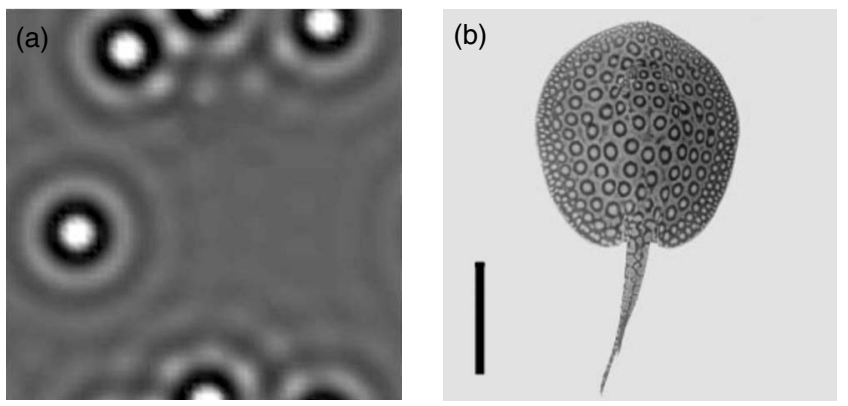

FIG. 1. (a) An example of pattern produced when two BVAM models are coupled together. The domain is square with side length of 120. Reproduced from [10]. "Copyright 2009 by the American Physical Society." (b) The skin pattern of the stingray Potamotrygon motoro. Scale bar of $10 \mathrm{~cm}$. 

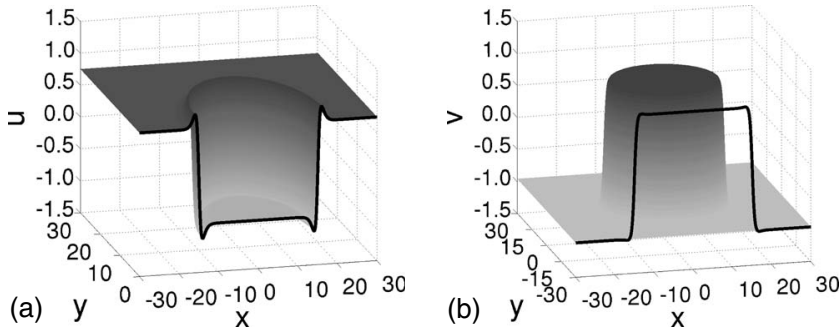

FIG. 2. (a) Cut away concentration of the stationary stable droplet, $u$, with the central profile emphasized in black. (b) Concentration of the stationary stable droplet, $v$, with central profile in black. $u$ and $v$ were found by solving Eqs. (3) and (4) with parameters $C=0.005, \quad D=0.516, \quad \eta=0.35, \quad h=-1.32, \quad a=0.05775$, and $b=$ -0.30525 .

stable homogeneous solutions, presenting a fixed radius (see Fig. 2). Spatially localized structures such as these have been the source of increased study in the field of nonlinear optics. Cavity solitons, which can form as one stable solution shrinks due to its embedding in the other, are common due to either the polarization symmetry or quadratic nature of the nonlinearity of many optical systems. Similarly, systems which show the stable coexistence of homogeneous and spatially modulated solutions can be used in the theory of optical coding and memory applications [11-13].

In a wide region of parameter space the walls (also known as fronts) travel with a constant velocity that depends on the asymmetry of the shape of the profile. From random initial conditions the number of fronts produced is such that a definite spatial scale appears. If too many fronts are created, such that their separation is less than the spatial scale, the excess are annihilated when the fronts collide, leaving free to move only those that have separation larger than the distance given by the scale [14]. The picture in two dimensions is more revealing: from initial random conditions the two phases immediately separate and the fronts start moving with a velocity that now depends also on the local curvature. The points where the curvature changes sign are then stationary and spiral movement of rotating stripes of constant width is observed (Fig. 3). In a different region of parameter space the velocity of the fronts becomes zero and a stationary labyrin- thine pattern settles in. Finally, when one changes the parameters around this region, one of the phases is more abundant than the other. The fronts start curling and closing the regions occupied by the minority phase, droplets are formed and some of the small ones disappear, while a number of the large ones shrink to a given radius that depends on the parameters of the model (see Fig. 2). In all other situations, fronts connecting two nonequivalent homogeneous states move in such a way that the more stable state annihilates the other [15].

In the Ginzburg-Landau system these droplets "appear close to the modulational instability of a flat domain wall and balance the curvature-driven shrinking of a domain with the growth due to the instability of tightly curved fronts" (p. 238 in [16]). Droplets are related to solitons and other local structures such as nuclei, although their structure is fundamentally different. Nuclei are never stable. They occur when the inner solution of a circular domain is favored. Thus, a structure starting with a large radius will grow to infinity [17]. The stability of the dark ring cavity solitons comes from the interacting oscillatory tails $[13,18]$. Whereas in the symmetric system the stability of the droplet is dependent on the curvature, we will show that, in the more general asymmetric case, the existence and stability of the droplets also depend crucially on the size of the asymmetry. Due to its existence depending on curvature, the state associated with stable droplets cannot exist in one dimension. Thus, we believe that, although we must turn to numerical simulation in the end, the analysis gives us insights into the structure, stability, and reality of these patterns. To analyze the dynamics we will extend the methods developed by Gomila et al. $[19,20]$ while examining the evolution of domain walls in nonlinear optical systems with a symmetric complex Ginzburg-Landau model. There it was shown quite generally that if:

(i) a system is invariant under translation and symmetric in its spatial variable (e.g., $x \rightarrow-x$ );

(ii) there exists a discrete symmetry that allows the existence of two and only two nonzero stable homogeneous solutions; and

(iii) in one dimension there exist Ising fronts that connect these solutions, then one is able to derive a formula for the radius of the droplets. This analysis can then be extended to

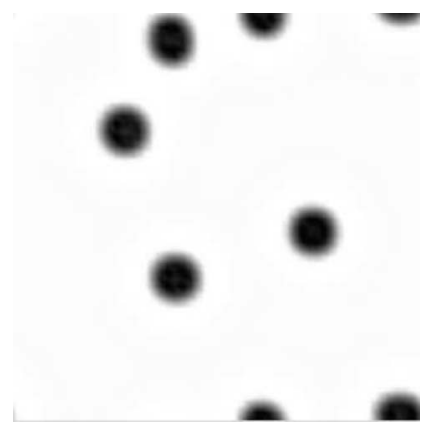

(a)

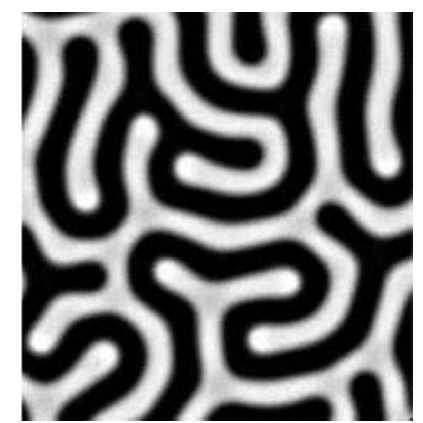

(b)

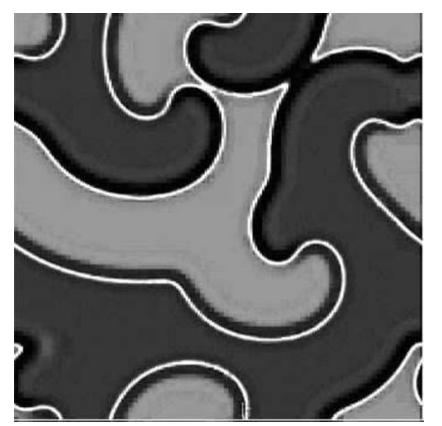

(c)

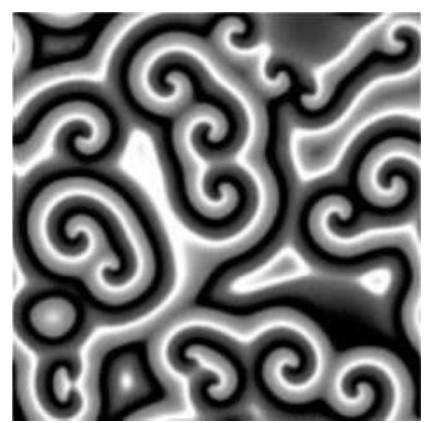

(d)

FIG. 3. Patterns obtained when $h \neq-1$ in Eqs. (3) and (4). (a) and (b) are stationary and found in region 2 of Fig. 5. (c) Traveling wave fronts, found in region 2. (d) Oscillating spiral patterns found on the border between regions 2 and 5 of Fig. 5 . All pattern peaks and troughs are contained in the region $[-2,2]$ and in each case the domain is square with side length of 254. Reproduced from [10]. "Copyright 2009 by the American Physical Society." 
encompass the case when the steady states are asymmetric [13].

In Sec. II we introduce the BVAM model and illustrate its various properties before deriving an approximate equation for the radius of the asymmetric droplets in Sec. III. At the end of Sec. III we turn to numerical methods to validate our analysis and comparisons are made in Sec. IV. Finally, in Sec. V we present conclusions.

\section{MODEL}

In this section we introduce the BVAM system and illustrate some of the many patterns that it can produce. The general form of a two-species reaction-diffusion system describing spatial and temporal variations of the concentrations $u$ and $v$, undergoing reactions described by the kinetic functions $G_{1}$ and $G_{2}$, with diffusivities $D_{u}$ and $D_{v}$, respectively, is [21]

$$
\begin{aligned}
& \frac{\partial u}{\partial t}=D_{u} \nabla^{2} u+G_{1}(u, v), \\
& \frac{\partial v}{\partial t}=D_{v} \nabla^{2} v+G_{2}(u, v) .
\end{aligned}
$$

The BVAM model is obtained by Taylor expanding the reaction kinetics [the nonlinear functions $G_{1}$ and $G_{2}$ in Eqs. (1) and (2)] around a stationary solution [9]. The terms of fourth and higher orders are neglected, partly for simplicity but also because analysis suggests that pattern selection between spots and stripes arises from an interplay between quadratic and cubic terms [22]. The resulting system can then be expressed in the following nondimensional form:

$$
\begin{aligned}
& \frac{\partial u}{\partial t}=D \nabla^{2} u+\eta\left(u+a v-C u v-u v^{2}\right), \\
& \frac{\partial v}{\partial t}=\nabla^{2} v+\eta\left(b v+h u+C u v+u v^{2}\right),
\end{aligned}
$$

where $C, D, a, b, h$, and $\eta$ are nondimensionalized parameters. We will consider the effect of parameters $C$ and $h$ on the stable droplets. The term $C$ adjusts the relative strength of the quadratic and cubic nonlinearities; this causes the system to alter its preference in terms of spotted or striped patterns. $C$ is also the symmetry-breaking parameter, such that when $C=0$ the spatially uniform steady states are symmetric about zero and asymmetric otherwise. The parameter $h$ is important as it controls the number of existing spatially uniform steady states. Specifically, if $h=-1$ then only the trivial stable steady state, $\left(u_{0}, v_{0}\right)=(0,0)$, exists. In order to minimize boundary effects we shall use periodic boundary conditions throughout this paper.

System (3) and (4) can be written in the more compact form,

$$
\frac{\partial \mathbf{u}}{\partial t}=\mathbf{D} \nabla^{2} \mathbf{u}+\mathbf{W}(\mathbf{u}, h)+C \mathbf{S}(\mathbf{u}),
$$

where

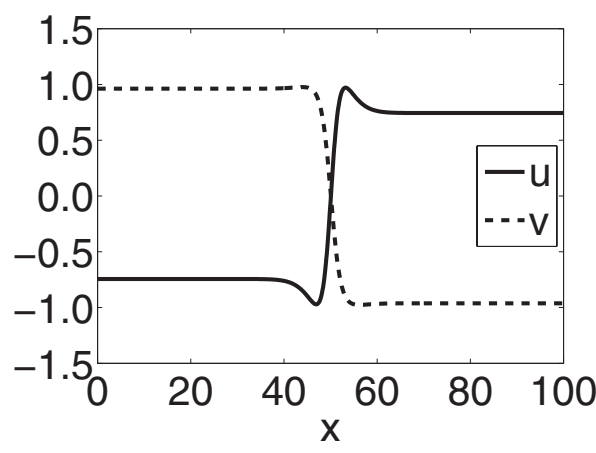

FIG. 4. Stationary symmetric structure profiles of $u$ (solid line) and $v$ (dashed line), obtained from Eqs. (3) and (4) in one dimension with parameters $C=0, \quad D=0.516, \quad \eta=0.35, \quad h=-1.32, a$ $=0.05775$, and $b=-0.30525$.

$$
\mathbf{u}=\left(\begin{array}{l}
u \\
v
\end{array}\right), \quad \mathbf{D}=\left(\begin{array}{ll}
D & 0 \\
0 & 1
\end{array}\right), \quad \mathbf{W}(\mathbf{u}, h)=\eta\left(\begin{array}{c}
u+a v-u v^{2} \\
b v+h u+u v^{2}
\end{array}\right),
$$

and

$$
\mathbf{S}(\mathbf{u})=u v\left(\begin{array}{c}
-1 \\
1
\end{array}\right)
$$

If $C=0$ and $h \neq-1$, there are three spatially uniform steady states, $\left(u_{0}, v_{0}\right)=(0,0)$ and $\left(u_{0}, v_{0}\right)_{ \pm}=v_{ \pm}(-g, 1)$, where

$$
v_{ \pm}= \pm \sqrt{\frac{b}{g}-h}, \quad g=\frac{a+b}{1+h} .
$$

We now note that the Laplacian makes the system invariant under translation and the transformation $\mathbf{u}(\mathbf{x}) \rightarrow \mathbf{u}(-\mathbf{x})$. Second, the forms of the kinetics are such that they cause the steady states to be equivalent under the discrete symmetry $v_{+} \rightarrow v_{-}$. Finally, as Ising fronts form between the two homogeneous solutions we can invoke the results of Gomila et al. [20]. The Ising walls that result when $C=0$ are perfectly symmetric in the sense that the area under any of the curves is exactly zero (Fig. 4). The interfaces can be shown to have a shape corresponding to

$$
u=-g v_{-} \tanh \left(\alpha_{1} x\right)+\alpha_{2} \sin \left(\alpha_{3} x\right) e^{-\alpha_{4}|x|},
$$

where $\left\{\alpha_{i}\right\}_{i=1}^{4}$ are constants which can be determined [14,23]. Observe that $\alpha_{4}=\sigma$ and $\alpha_{3}=\tau$ are the real and imaginary parts, respectively, of the eigenvalues of the linearized system about one of the nonzero critical points. $\alpha_{1}$ and $\alpha_{2}$ can be fitted numerically.

If $C \neq 0$ and $h \neq-1$,

$$
v_{ \pm}=\frac{-C \pm \sqrt{C^{2}+4\left(\frac{b}{g}-h\right)}}{2} .
$$

Thus, the nonzero steady states are no longer equivalent. In this case the Ising front can move as the asymmetry causes the system to favor one of the states.

Standard stability analysis [24] shows that the existence of nonzero fixed points causes the BVAM model to have a wide range of available patterns (see Fig. 3). For instance, if one fixes the value of $h$, the complex eigenvalues $(\omega=\sigma$ 


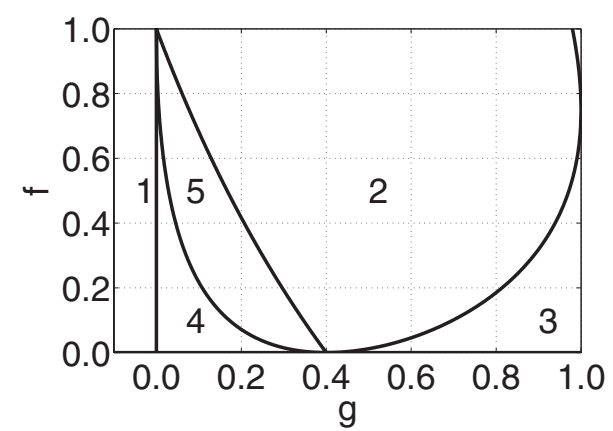

FIG. 5. Phase plane, in the absence of diffusion, in Eqs. (3) and (4). Parameters are $D=0, C=0$, and $h=-2.5$. The lines separating the regions are derived from the dispersion relation $\omega^{2}-\omega \operatorname{Tr}(\mathbf{J})$ $+\operatorname{Det}(\mathbf{J})=0$, where $\omega=\sigma_{j}+i \tau_{j}, j=1$ and 2 . In region $1, \sigma_{1}<0, \sigma_{2}$ $>0$, and $\tau=0$ (saddle points). In region $2, \sigma_{1,2}<0$ and $\tau \neq 0$ (oscillating stable points). In region $3, \sigma_{1,2}<0$ and $\tau=0$ (stable points). In region $4, \sigma_{1,2}>0$ and $\tau=0$ (both points are unstable). In region 5, $\sigma_{1,2}>0$ and $\tau \neq 0$ (oscillating unstable points).

$+i \tau)$ can be examined in the plane $(g, f)$, where $f=b / g-h$ $=v_{ \pm}^{2}$. The $(g, f)$ space can be divided into five regions of different dynamics shown in Fig. 5. Detailed analyses of the model in these regions have been published elsewhere $[10,14,25]$.

Specifically, we note that starting from a moving front pattern, as $C$ is increased we see a transition through labyrinthine stationary patterns to disordered circular domains of fixed radius. This transition is not sharp and one could have a combination of stripes and spots (see Fig. 5 in [10]).

One specific application of the model in the stable droplet region has been the pigmentation patterning in freshwater stingrays of the family Potamotrygonidae. The patterns of the potamotrygonid species are mostly established by birth or shortly thereafter. They have highly intricate dorsal color patterns, which do not look like simple Turing patterns. These patterns include variously shaped spots [26]. By weakly coupling two sets of BVAM equations together not only we can get a good qualitative comparison between the stingray Potamotrygon motoro and the simulations (Fig. 1) but also we can account for the differences between genders and the change in patterning that occurs as the stingray matures [10].

\section{ANALYSIS OF DROPLETS IN THE BVAM MODEL}

To analyze the droplets we must first reduce system (5) using assumptions that allow us to keep only the crucial dynamics of the droplets and thus remove the dark ring cavity solitons from the system. We construct a coordinate system $(w, s)$, which moves with the front. $w$ is the normal coordinate to the front and $s$ is the arc length along the front (see Fig. 6). The change of coordinates is then defined as

$$
\mathbf{x}(w, s, t)=\mathbf{X}(s, t)+w \hat{\mathbf{n}}(s, t),
$$

where $\mathbf{x}$ is the position vector in the reference frame and $\mathbf{X}$ is the position vector of the line front in the reference frame. The vector $\hat{\mathbf{n}}$ is the unit normal vector to the line front in the

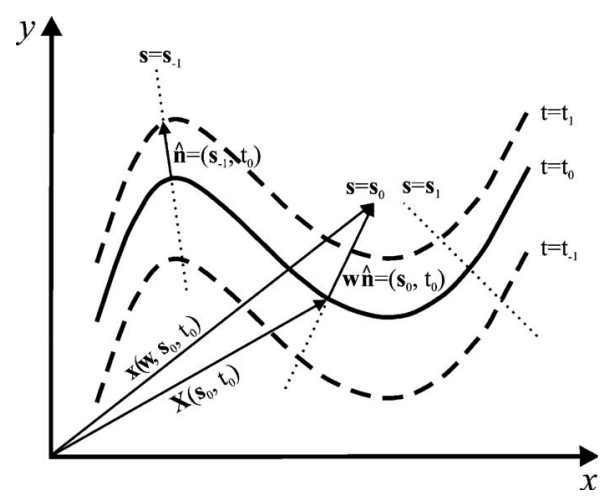

FIG. 6. Illustration of the new coordinate variables given by Eq. (9). $\mathbf{x}$ is the position vector in the reference frame and $\mathbf{X}$ is the position vector of the line front. The vector $\hat{\mathbf{n}}$ is the unit normal vector to the line front, $w$ is the normal coordinate to the front, and $s$ is the arc length along the front. The various $s_{i}$ are illustrative points along the front. Similarly, the $t_{i}$ denotes the evolution of the system at different time points.

reference frame. Under this coordinate transformation, Eq. (5) can be written in the form

$$
\begin{aligned}
\frac{\partial \mathbf{u}}{\partial t}= & \mathbf{D} \frac{\partial^{2} \mathbf{u}}{\partial w^{2}}+\left(\hat{n}_{v} I+\frac{\kappa \mathbf{D}}{1+w \kappa}\right) \frac{\partial \mathbf{u}}{\partial w}+\frac{\kappa^{2} \mathbf{D}}{(1+w \kappa)^{2}} \frac{\partial^{2} \mathbf{u}}{\partial \theta^{2}}+\mathbf{W}(\mathbf{u}, h) \\
& +C \mathbf{S}(\mathbf{u}),
\end{aligned}
$$

where $\kappa=\nabla \cdot \hat{\mathbf{n}}$ is the curvature, $\hat{n}_{v}=\partial \mathbf{X} / \partial t \cdot \hat{\mathbf{n}}$ is the normal front velocity, and $\theta=\kappa s$ is the azimuthal angle. Since we are looking for a circular droplet structure with radius $R$, these can be translated to $\hat{n}_{v}=\dot{R}$ and $\kappa=1 / R$.

To enable us to analyze the equation effectively we make the following assumptions which will be justified later:

(1) in the moving frame of the Ising front, the droplet structure is stationary;

(2) the stable droplet has no $\theta$ (azimuthal angle) dependence;

(3) the radius of the stable droplet, $R$, is large, $R \gg 1$;

(4) the asymmetry is small $(C \approx 0)$ but different from zero; and

(5) we are able to perturb around the stationary profile of the one-dimensional symmetric system, $\mathbf{u}_{0}$, using a slightly curved front of the form $\mathbf{u}=\mathbf{u}_{0}(w)+\mathbf{u}_{1}(w, s, t)$, where $\left|\mathbf{u}_{1}\right|$ $\ll\left|\mathbf{u}_{0}\right|$.

Assumption 1 assures us that we are looking for stationary structures, so we can equate the time derivative to zero. By considering Fig. 7 we see the differences between the soliton structure and droplet profiles. The stable droplets have a larger radius and are more cylindrical in structure than the soliton structures. Assumptions 2 and 3 keep these important properties in the simplified model and are justified by considering Fig. 2. Since $M$ is only singular when $C=0, C$ is allowed to be small but nonzero. This avoids the singularity during the derivation but causes $M$ to be approximately singular, thus justifying assumption 4 . Finally, assumption 5 is critical in enabling us to reduce the dimension from two to one by allowing us to treat the domain walls as essentially 

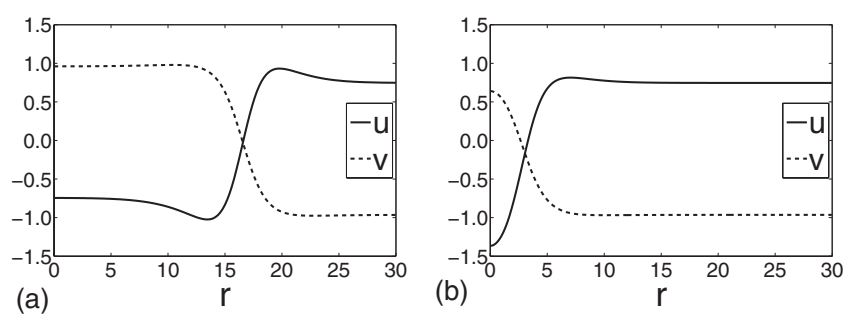

(b)

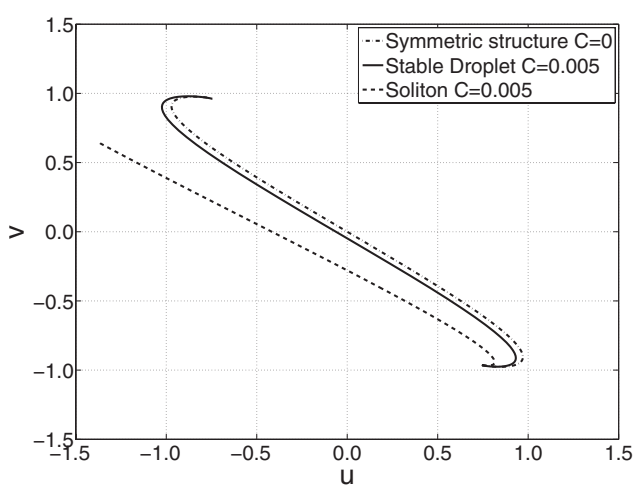

FIG. 7. (a) Profiles of droplet structures $u$ (solid line) and $v$ (dashed line). (b) Profiles of soliton structures $u$ (solid line) and $v$ (dashed line). (c) Phase plane plot. The dashed-dotted trajectory is the solution to Eq. (18). The solid trajectory is the droplet profile. The dashed trajectory is the soliton profile. The latter two solutions were obtained by solving Eq. (21) with different initial conditions. In (a) and (b) $C=0.005$, the values of $C$ in (c) are noted in the figure. Other parameters are $D=0.516, \quad \eta=0.35, h=-1.32, a$ $=0.05775$, and $b=-0.30525$.

one-dimensional with the curvature as a small perturbation around this.

By considering Eq. (10) and applying assumptions 1-5 we see that the leading-order term, $\mathbf{u}_{0}(w)$, will be the solution to Eq. (18). Further, after the application of the assumptions and Taylor's theorem, the correction term, $\mathbf{u}_{1}$, will satisfy

$$
0=\mathbf{D} \frac{\partial^{2} \mathbf{u}_{1}}{\partial w^{2}}+\left(\dot{R} I+\frac{1}{R} \mathbf{D}\right) \frac{\partial \mathbf{u}_{0}}{\partial w}+\mathbf{J}\left(\mathbf{u}_{0}, h\right) \mathbf{u}_{1}+C \mathbf{S}\left(\mathbf{u}_{0}\right) .
$$

We let

$$
\begin{gathered}
M=\mathbf{D} \frac{\partial^{2}}{\partial w^{2}}+\mathbf{J}\left(\mathbf{u}_{0}, h\right) \\
\Rightarrow M \mathbf{u}_{1}=-C \mathbf{S}-\left(\dot{R} I+\frac{1}{R} \mathbf{D}\right) \frac{\partial \mathbf{u}_{0}}{\partial w},
\end{gathered}
$$

where $\mathbf{J}$ is the standard Jacobian of the kinetic terms (W) evaluated at $\left(\mathbf{u}_{0}, h\right)$ and $\mathbf{S}=\mathbf{S}\left(\mathbf{u}_{0}\right)$ is a constant. We note that $M$ is singular as Eq. (5) is translationally invariant. Hence, there exists a null vector, $\mathbf{e}_{0}=\partial \mathbf{u}_{0} / \partial w$, such that $M \mathbf{e}_{0}=0$. We can now use the Fredholm alternative [27] to produce a solvability criterion. We define the inner product to be $\langle\mathbf{a}, \mathbf{b}\rangle$ $=\int_{-\infty}^{\infty} \mathbf{a} \cdot \mathbf{b} d w$ and $\mathbf{a}_{0}$ to be the null mode of the adjoint operator $M^{T}$, e.g., $M^{T} \mathbf{a}_{0}=0$. Thus, Eq. (13) has a solution if and only if

$$
\begin{aligned}
& 0=\left\langle\mathbf{a}_{0},-C \mathbf{S}-\left(\dot{R} I+\frac{1}{R} \mathbf{D}\right) \frac{\partial \mathbf{u}_{0}}{\partial w}\right\rangle \\
& \Rightarrow \dot{R}=-C \frac{\left\langle\mathbf{a}_{0}, \mathbf{S}\right\rangle}{\left\langle\mathbf{a}_{0}, \mathbf{e}_{0}\right\rangle}-\frac{1}{R} \frac{\left\langle\mathbf{a}_{0}, \mathbf{D} \mathbf{e}_{0}\right\rangle}{\left\langle\mathbf{a}_{0}, \mathbf{e}_{0}\right\rangle} .
\end{aligned}
$$

Next define

$$
\nu_{0}(h)=-\frac{\left\langle\mathbf{a}_{0}, \mathbf{S}\right\rangle}{\left\langle\mathbf{a}_{0}, \mathbf{e}_{0}\right\rangle}, \quad \gamma(h)=\frac{\left\langle\mathbf{a}_{0}, \mathbf{D} \mathbf{e}_{0}\right\rangle}{\left\langle\mathbf{a}_{0}, \mathbf{e}_{0}\right\rangle} .
$$

Depending on the signs of the various terms the equilibrium radius, $R_{0}$, is given by

$$
R_{0}=\frac{\gamma(h)}{\nu_{0}(h)}=-\frac{1}{C} \frac{\left\langle\mathbf{a}_{0}, \mathbf{D} \mathbf{e}_{0}\right\rangle}{\left\langle\mathbf{a}_{0}, \mathbf{S}\right\rangle}=-\frac{1}{C} \frac{\int_{-\infty}^{\infty} \mathbf{a}_{0} \cdot \mathbf{D} \mathbf{e}_{0} d w}{\int_{-\infty}^{\infty} \mathbf{a}_{0} \cdot \mathbf{S} d w} .
$$

We will be interested in the parameter $\gamma(h)$ later, as $\gamma=0$ is the criterion for the modulational instability of a flat front. We define $h_{c}$ to be such that $\gamma\left(h_{c}\right)=0$. Note also that $\left\langle\mathbf{a}_{0}, \mathbf{e}_{0}\right\rangle=0$ at an Ising-Bloch transition [28] so we consider only parameter regions far away from this bifurcation point. As can be seen from Fig. 7 the profile changes from the positive solution to the negative solution over a short interval so we define the radius to be the point where the profile of $v$ crosses zero.

Now that we have derived a formula for the theoretical radius we must turn to computation in order to evaluate $\mathbf{a}_{0}$ and $\mathbf{e}_{0}$. We must first compute $\mathbf{u}_{0}$; the solution of the onedimensional, stationary, symmetric equation

$$
F=\left(\begin{array}{c}
D \frac{d^{2} u}{d x^{2}} \\
\frac{d^{2} v}{d x^{2}}
\end{array}\right)+\eta\left(\begin{array}{c}
u+a v-u v^{2} \\
b v+h u+u v^{2}
\end{array}\right) \equiv 0 .
$$

In order to compute the solution, we apply a NewtonRaphson method [29] in Eq. (18) using its Jacobian,

$$
M=\left(\begin{array}{c}
D \frac{d^{2} u}{d x^{2}} \\
\frac{d^{2} v}{d x^{2}}
\end{array}\right)+\eta\left(\begin{array}{cc}
1-v^{2} & a-2 u v \\
h+v^{2} & b+2 u v
\end{array}\right) .
$$

This is achieved by discretizing $F$ and $M$ using a secondorder finite difference spatial operator to approximate the derivatives. This creates banded matrices that can be solved for efficiently [30]. If the system is initialized with a solution that is close enough to the droplet wall form the iterates will tend to converge on a solution of Eq. (18) which is $\mathbf{u}_{0}$ $=\left(u_{0}, v_{0}\right)$. Due to the walls having the explicit form shown in Eq. (7), a tanh function was used as the initial seed. Finally, $\mathbf{e}_{0}$ is calculated using the definition 


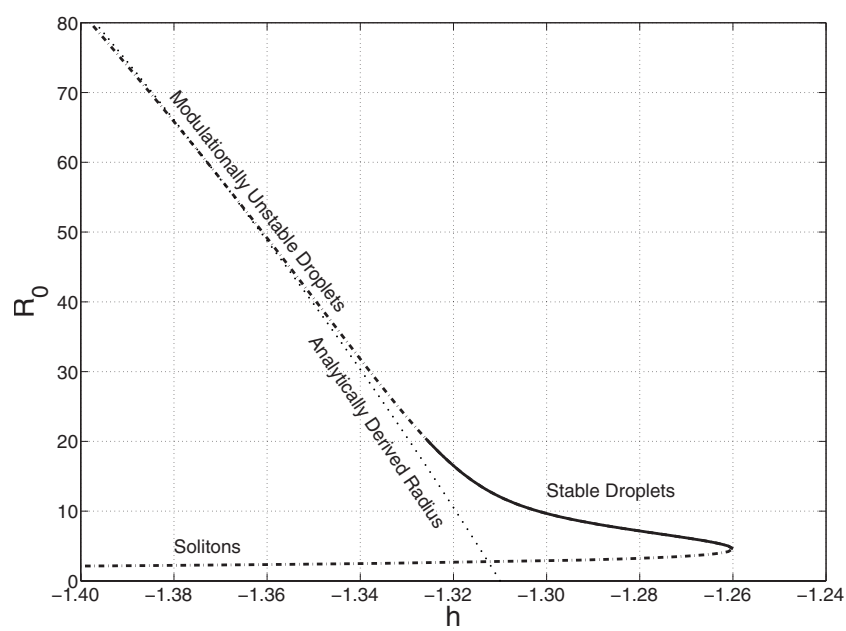

FIG. 8. Comparison of the computed steady-state radius of the perturbed system with the analytically derived radius attained from one-dimensional Eqs. (18) and (21). The dashed line indicates instability to either radial or azimuthal perturbations. The solid line indicates a radius stable to radial and azimuthal perturbations. The dotted line is the theoretical prediction from Eq. (17) with $C$ $=0.005$ and all other parameters as in Fig. 7. The stability of Eqs. (3) and (4) with a two-dimensional Laplacian was found by using a finite element method on a mesh of 9440 elements. Through a bisection parameter search the stability disappears at $h_{d}=-1.326$, correct to three decimal places.

$$
\mathbf{e}_{0}=\frac{\partial \mathbf{u}_{0}}{\partial w}=\left(\begin{array}{c}
\frac{d u_{0}}{d x} \\
\frac{d v_{0}}{d x}
\end{array}\right)
$$

and $\mathbf{a}_{0}$ is calculated by finding the null vector of $M^{T}$ (which can be done using any number of numerical techniques $[31,32])$.

To compare the approximation with the actual computed radius of the droplets we use the Newton-Raphson method on the radial, angular independent, stationary, asymmetric equation,

$$
\begin{aligned}
\hat{F} & =\left(\begin{array}{c}
D\left(\frac{d^{2}}{d r^{2}}+\frac{1}{r} \frac{d}{d r}\right) u \\
\left(\frac{d^{2}}{d r^{2}}+\frac{1}{r} \frac{d}{d r}\right) v
\end{array}\right)+\eta\left(\begin{array}{c}
u+a v-u v^{2} \\
b v+h u+u v^{2}
\end{array}\right)+\eta C u v\left(\begin{array}{c}
-1 \\
1
\end{array}\right) \\
& \equiv 0
\end{aligned}
$$

with Jacobian

$$
\begin{aligned}
\hat{M}= & \left(\begin{array}{c}
D\left(\frac{d^{2}}{d r^{2}}+\frac{1}{r} \frac{d}{d r}\right) u \\
\left(\frac{d^{2}}{d r^{2}}+\frac{1}{r} \frac{d}{d r}\right) v
\end{array}\right)+\eta\left(\begin{array}{cc}
1-v^{2} & h+v^{2} \\
a-2 u v & b+2 u v
\end{array}\right) \\
& +\eta C\left(\begin{array}{cc}
-v & -u \\
v & u
\end{array}\right) .
\end{aligned}
$$

By solving the stationary equation [Eq. (21)], we are able to
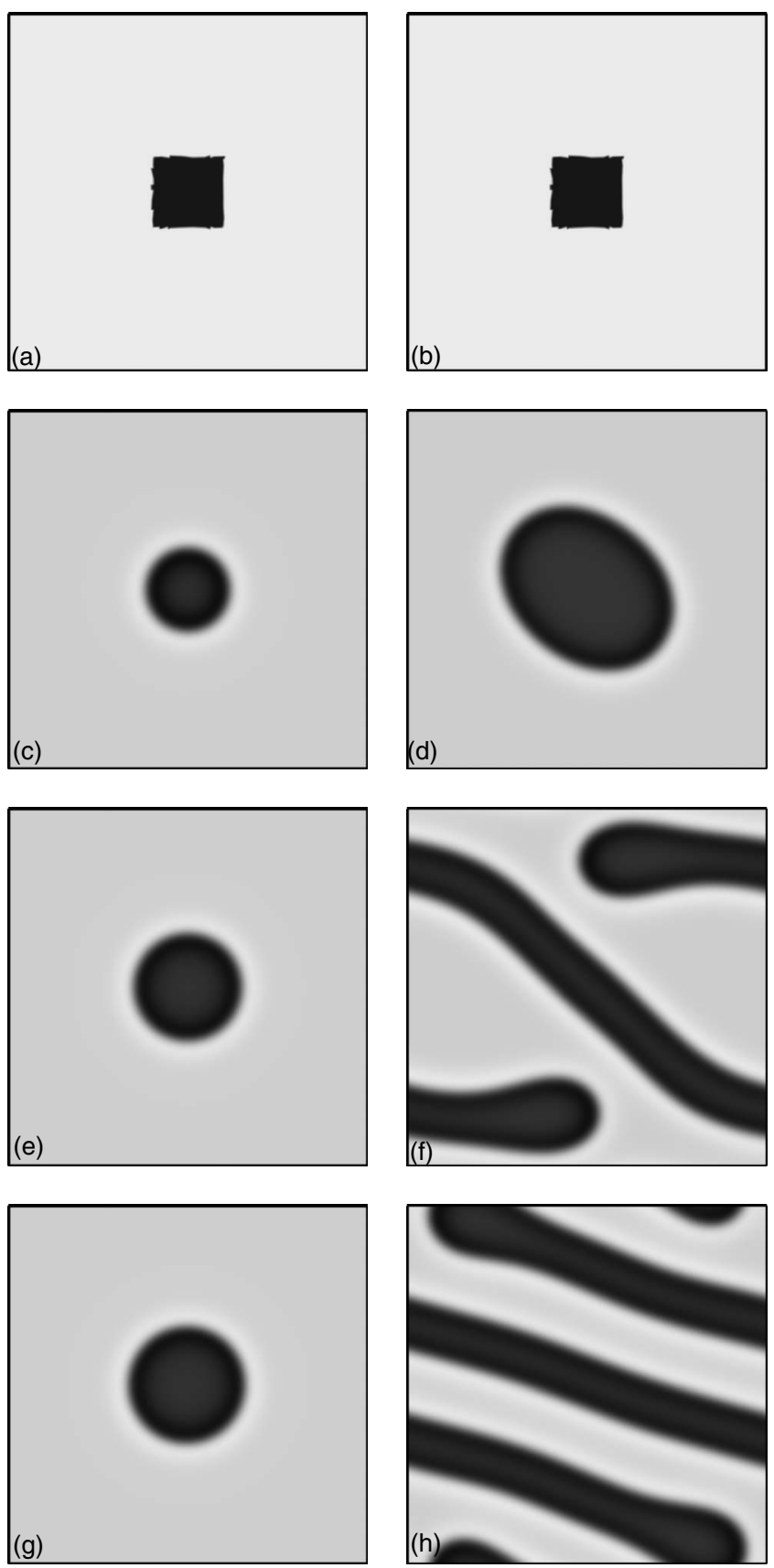

FIG. 9. Time series for the evolution of a square initial condition. The left column has $h=-1.32$ and so is in the stable region; thus, the system reaches a final droplet steady pattern. The times shown are $t=0,100,10^{4}$, and $10^{5}$. The right column has $h=-1.33$ and so is in the unstable region; thus, it produces a labyrinthine steady state. The times shown are $t=0,10^{5}, 2 \times 10^{5}$, and $10^{6}$. Other parameters are $D=0.516, \eta=0.35, C=0.005, a=0.05775$, and $b=$ -0.30525 . The gray scale is from -1.2 (darkest) to 1.2 (lightest) and the domain is $[-50,50] \times[-50,50]$.

find both stable and unstable structures. The radial stability of the structure is found naturally by considering the eigenvalues of the operator $\hat{M}$, which is the Jacobian of the system and is available quite readily due to its involvement in the Newton-Raphson method [29]. 

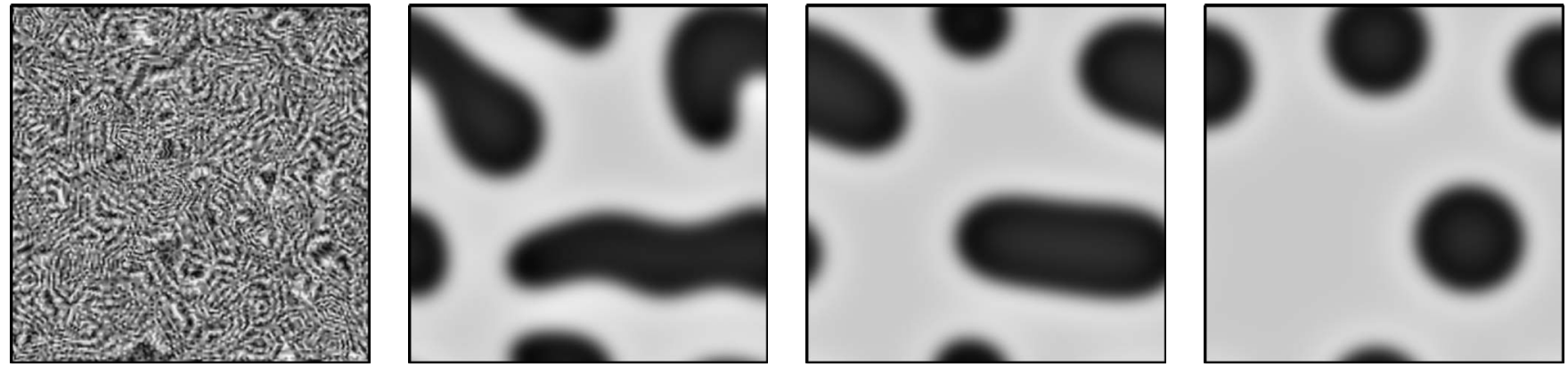

FIG. 10. Time series for the evolution of pseudorandom initial conditions to stable droplets. The times shown are $t=0,100,10^{3}$, and $10^{4}$. Parameters $D=0.516, \eta=0.35, C=0.005, h=-1.3, a=0.05775$, and $b=-0.30525$. The gray scale is from -1.4 (darkest) to 1.4 (lightest) and the domain is $[-50,50] \times[-50,50]$.

\section{RESULTS}

Observe in Fig. 8 that when the radius is large there is a good agreement between the computed one-dimensional radius from Eq. (21) and the analytically derived radius gained from the perturbation analysis in Eq. (17). However, the approximation breaks down for $h>-1.32$. The reason for this breakdown is due to assumption 3 , the assumption of shallow curvature, or large radius, becoming invalid for $h \approx$ -1.32 . For $h_{c}$ defined as the point of modulational instability, as in Sec. III $\left[\gamma\left(h_{c}\right)=0\right]$, then to three decimal places this can be calculated by a bisection search as $h_{c}=-1.310$. This implies that when $C=0$ stable droplets cannot exist for $h<h_{c}$ as the states are completely symmetric. In this situation the most abundant phase will tend to wipe the other out leaving a homogeneous steady state. Although the symmetric system cannot have stable droplets for $h<h_{c}$, when $C>0$ we see from Eq. (15) that the asymmetry is able to stabilize the droplets for a larger parameter region so, as Fig. 8 clearly shows, stable droplets exist for $h<h_{c}$. For $h<h_{c}(\gamma<0)$, both droplet and local structure (dark ring cavity soliton) profiles exist in the radial stationary equation [Eq. (21)]. Further, the one-dimensional analysis predicts that all droplet profiles should be stable to radial perturbations as $\nu_{0}<0$ throughout this parameter region. This is corroborated by the negative eigenvalues of the Jacobian $\hat{M}$.

It should be noted that although all the droplet profiles are stable to radial perturbations, modulational instabilities of their circular wall in the azimuthal direction can occur. In two-dimensional simulations (Fig. 9) the stability of the droplets disappears somewhere between $h=-1.33$ and -1.32 . To simulate the two-dimensional equations we used a finiteelement method on a mesh of 9440 elements and through a bisection parameter search we found the boundary of stability to be $h=-1.326=h_{d}$ correct to three decimal places. For $h>h_{d}$ the droplets were able to stabilize [Fig. 9(g)]; for $h$ $<h_{d}$ the droplets succumbed to azimuthal instabilities and broke down [Fig. 9(d)]. The local structures were never seen to be stable, corroborating the stability information gained from the asymmetric radial equation.

Within the region $h_{d}<h<-1.26$ the droplets are not only stable but also appear to be very robust. Figure 10 shows that, from pseudorandom initial conditions $[u(x, y, 0)$ $=\sin (90000 x+9000 y)+\cos (239845 x y), \quad v(x, y, 0)=\sin (45$
$678 x+4659 y)-\cos (379586 x y)-\sin (20000 x+88000 y)]$, the system exhibits stable droplets. The only other pattern observed to be viable in this region from pseudorandom initial conditions is stripes (results not shown). These stripes are not the labyrinthine patterns we would normally consider. The stripes are able to form due to the periodic boundary conditions as they form a continuous band through the domain producing a structure with no end. If the stripes are cut they shrink to droplets as they are unable to support unjoined ends, unlike the curved heads seen in Figs. 9(f) and 9(h) which labyrinthine patterns can produce. Zero flux boundary conditions have the potential to stop such stripes forming, leaving stable droplets as the only pattern available.

\section{CONCLUSION}

The BVAM model examined here presents the Turing, Hopf, and Turing-Hopf bifurcations when $h \neq-1$. This richness of behavior allows us to gain insight into the mechanisms of pattern formation and selection in a wide variety of problems. In particular, in the bistable regime it is possible to obtain stable cylindrical structures with large radius. An important fact underlying this phenomenon is that $\nu_{0}<0$ throughout the chosen parameter region. If, instead $\nu_{0}>0$, the structures would not have been stable droplets, they would have been unstable nuclei.

Observe in Figs. 9 and 10 that the stable structures evolve into their stationary forms much quicker than the unstable structures. The stable structures form on a time scale of order $10^{4}$ (nondimensional units), whereas the unstable structures form on a longer time scale of order $10^{6}$. It was observed that, starting from nonrandom initial conditions, such as a square or circle, the unstable structures first form a circle which would be stable for quite some time, then quickly rearrange into another metastable structure, such as a spacefilling square. Finally, after another length of time, the pattern reorganizes very quickly to a labyrinthine pattern.

As the patterns take such a long time to form in the nondimensional system, we must determine if these time scales are consistent with the notion of the BVAM mechanism as a potential candidate for biological patterning. To answer this we must first consider the original dimensional form of the BVAM model [25],

$$
u_{t}=D \delta \nabla^{2} u+\alpha u\left(1-r_{1} v^{2}\right)+v\left(\tau-r_{2} u\right),
$$




$$
v_{t}=\delta \nabla^{2} v+v\left(\beta+\alpha r_{1} v u\right)+u\left(\gamma-r_{2} v\right) .
$$

To nondimensionalize we let $u=U \hat{u}, v=V \hat{v}, t=T \hat{t}$, and $x$ $=L \hat{x}$, where the capital letters are the dimensional scales and the hatted variables are nondimensional variables. After rearrangement we obtain

$$
a=\frac{\tau}{\alpha}, \quad b=\frac{\beta}{\alpha}, \quad h=\frac{\gamma}{\alpha}, \quad C=\frac{r_{2}}{\alpha \sqrt{r_{1}}}, \quad \eta=\frac{L^{2} \alpha}{\delta}, \quad T=\frac{L^{2}}{\delta} .
$$

At birth, stingrays range from 10 to $100 \mathrm{~mm}$ in length [33]. Since $\hat{x}=100$, we use $L=10^{-2} \mathrm{~cm}$. For the diffusivity $\delta$ $=10^{-6}-10^{-7} \mathrm{~cm}^{2} / \mathrm{s}$ are biologically consistent values [34,35], giving $T=10^{2}-10^{3} \mathrm{~s}$, which implies $t=10^{6}-10^{7} \mathrm{~s}$ $\approx 12-115$ days. Since the gestation period of the stingrays varies between three and nine months [36], stable droplet formation through a bistable system is therefore a viable mechanism of pattern formation.

We have studied the effect of a symmetry-breaking parameter on the existence and stability of stable droplets and local structures. The strength of this technique lies in the fact that although the droplets can only exist in two dimensions, we can still use a one-dimensional analysis to derive an asymptotic approximation for the radius. From Eq. (17) we can see that the form of the asymmetry, $S$, the size of asymmetry, $C$, and the nondimensionalized diffusion coefficient, $D$, are all critical factors when it comes to determining the size and stability of the droplets.

We have shown that droplet and local structure profiles are both solutions to the stationary radial equations given by Eq. (21). Further, the droplet profiles are always stable to radial perturbations whereas the local structures are always unstable to radial perturbations. In two dimensions the parameter range of existence is much smaller due to the destabilizing effects of curvature on the droplet structure. However, in the region of existence, not only are the droplets stable but they also appear to be quite robust.

The first-order perturbation analysis gives a good approximation for $-1.4<h<-1.32$ when $C=0.005$. As $h \rightarrow-1.32$, the assumption of the droplets having large radius begins to break down; thus, the reduction of the model based on gently curved fronts is no longer valid. Due to the reduction of the model from two dimensions to one, the theory is currently unable to predict when curvature effects become important and this extension is currently in progress.

\section{ACKNOWLEDGMENTS}

T.E.W. would like to thank Willie Firth and Damià Gomila for their helpful discussions and clarifications and Stephen and Cassandra Edwards for their information on breeding stingrays and their newborn. T.E.W. would also like to thank the EPSRC for support. R.E.B. would like to thank Research Councils U.K. and St. Hugh's College, Oxford for support. P.K.M. was partially supported by a Royal SocietyWolfson Merit Award. R.A.B. would like to thank CONACyT for financial support through Project No. 79641. J.L.A. would like to thank CONACyT for research Grant No. 50368 and DGAPA-UNAM for sabbatical grants. R.A.B. and J.L.A. are grateful for the hospitality received at the Centre for Mathematical Biology, Mathematical Institute, University of Oxford.
[1] P. DeKepper, V. Castets, E. Dulos, and J. Boissonade, Physica D 49, 161 (1991).

[2] D. G. Míguez, M. Dolnik, A. P. Muñuzuri, and L. Kramer, Phys. Rev. Lett. 96, 048304 (2006).

[3] A. M. Turing, Philos. Trans. R. Soc. London, Ser. B 237, 37 (1952).

[4] A. Gierer and H. Meinhardt, Biol. Cybern. 12, 30 (1972).

[5] S. Kondo and R. Asai, Nature (London) 376, 765 (1995).

[6] S. Sick, S. Reinker, J. Timmer, and T. Schlake, Science 314, 1447 (2006).

[7] S. S. Liaw, C. C. Yang, R. T. Liu, and J. T. Hong, Phys. Rev. E 64, 041909 (2001).

[8] H. Meinhardt, P. Prusinkiewicz, and D. R. Fowler, The Algorithmic Beauty of Sea Shells (Springer Verlag, Berlin, 2003).

[9] R. A. Barrio, C. Varea, J. L. Aragón, and P. K. Maini, Bull. Math. Biol. 61, 483 (1999).

[10] R. A. Barrio, R. E. Baker, B. Vaughan, Jr., K. Tribuzy, M. R. de Carvalho, R. Bassanezi, and P. K. Maini, Phys. Rev. E 79, 031908 (2009).

[11] W. J. Firth and G. K. Harkness, Asian J. Phys. 7, 665 (1998).

[12] W. J. Firth and A. J. Scroggie, Phys. Rev. Lett. 76, 1623 (1996).

[13] D. Gomila, P. Colet, G. L. Oppo, and M. S. Miguel., J. Opt. B:
Quantum Semiclassical Opt. 6, S265 (2004).

[14] C. Varea, D. Hernández, and R. A. Barrio, J. Math. Biol. 54, 797 (2007).

[15] D. Michaelis, U. Peschel, F. Lederer, D. V. Skryabin, and W. J. Firth, Phys. Rev. E 63, 066602 (2001).

[16] D. Gomila, P. Colet, M. San Miguel, A. J. Scroggie, and G. L. Oppo, IEEE J. Quantum Electron. 39, 238 (2003).

[17] J. D. Gunton, M. San Miguel, and P. S. Sahni, Phase Transitions and Critical Phenomena (Academic, London, 1983).

[18] G. L. Oppo, A. J. Scroggie, and W. J. Firth, J. Opt. B: Quantum Semiclassical Opt. 1, 133 (1999).

[19] D. Gomila, Ph.D. thesis, Universitat de les Illes Balears, 2003.

[20] D. Gomila, P. Colet, G. L. Oppo, and M. S. Miguel, Phys. Rev. Lett. 87, 194101 (2001).

[21] J. D. Murray, Mathematical Biology I: An Introduction, 3rd ed. (Springer, New York, 2003), Vol. 1.

[22] B. Ermentrout, Proc. R. Soc. Lond. A 434, 413 (1991).

[23] P. Coullet, C. Elphick, and D. Repaux, Phys. Rev. Lett. 58, 431 (1987).

[24] D. W. Jordan and P. Smith, Mathematical Techniques: An Introduction for the Engineering, Physical, and Mathematical Sciences (Oxford University Press, Oxford, 2002).

[25] T. Leppänen, Ph.D. thesis, Helsinki University of Technology, 
2004.

[26] M. R. de Carvalho, N. R. Lovejoy, and R. S. Rosa, Check List of the Freshwater Fishes of South and Central America, Chapter Family Potamotrygonidae (River Stingrays) (EDIPUCRS, Porto Alegre, 2003), p. 22.

[27] J. P. Keener, Principles of Applied Mathematics (Westview Press, Boulder, CO, 2000).

[28] D. V. Skryabin, A. Yulin, D. Michaelis, W. J. Firth, G. L. Oppo, U. Peschel, and F. Lederer, Phys. Rev. E 64, 056618 (2001).

[29] E. Süli and D. F. Mayers, An Introduction to Numerical Analysis (Cambridge University Press, Cambridge, 2003).

[30] J. M. McSloy, Ph.D. thesis, University of Strathclyde, 2002.
[31] C. B. Moler and G. W. Stewart, SIAM Journal on Numerical Analysis 10, 241 (1973).

[32] C. F. Van Loan, SIAM Journal on Numerical Analysis 12, 819 (1975).

[33] T. B. Thorson, J. K. Langhammer, and M. I. Oetinger, Environ. Biol. Fishes 9, 3 (1983).

[34] E. A. Dawes, Quantitative Problems in Biology (Longman, London, 1956).

[35] R. Dillon, C. Gadgil, and H. G. Othmer, Proc. Natl. Acad. Sci. U.S.A. 100, 10152 (2003).

[36] P. Charvet-Almeida, M. L. G. Araújo, and M. P. Almeida, J. Northwest Atl. Fish. Sci. 35, 165 (2005). 